\title{
Dynamic assessment of venous anatomy and function in neurosurgery with real-time intraoperative multimodal ultrasound: technical note
}

\author{
Francesco Prada, MD, ${ }^{1,2}$ Massimiliano Del Bene, MD, ${ }^{1,3}$ Giovanni Mauri, MD, ${ }^{4}$ \\ Massimo Lamperti, MD, ${ }^{5}$ Davide Vailati, MD, ${ }^{6}$ Carla Richetta, MD, ${ }^{7}$ Marco Saini, MD, ${ }^{1}$ \\ Davide Santuari, MD, ${ }^{8}$ M. Yashar S. Kalani, MD, PhD, ${ }^{2}$ and Francesco DiMeco, MD1,9
}

${ }^{1}$ Department of Neurosurgery, Fondazione IRCCS Istituto Neurologico C. Besta, Milan, Italy; ${ }^{2}$ Department of Neurological Surgery, University of Virginia Health Science Center, Charlottesville, Virginia; Departments of ${ }^{3}$ Experimental Oncology and ${ }^{4}$ Radiology, European Institute of Oncology, Milan, Italy; ${ }^{5}$ Anesthesiology Unit, Cleveland Clinic, Abu Dhabi, United Arab Emirates; ${ }^{6}$ Anesthesiology Unit, Ospedale di Circolo di Melegnano, Presidio di Vizzolo Predabissi, Milan, Italy; ${ }^{7}$ Department of Neurosurgery, Sourasky Medical Center, Tel Aviv, Israel; ${ }^{8}$ Department of Vascular Surgery, Ospedale S. Carlo, Milan, Italy; and 9Department of Neurological Surgery, Johns Hopkins Medical School, Baltimore, Maryland

\begin{abstract}
The relevance of the cerebral venous system is often underestimated during neurosurgical procedures. Damage to this draining system can have catastrophic implications for the patient. Surgical decision-making and planning must consider each component of the venous compartment, from the medullary draining vein to the dural sinuses and extracranial veins. Intraoperative ultrasound (ioUS) permits the real-time study of venous compartments using different modalities, thus allowing complete characterization of their anatomical and functional features. The B-mode (brightness mode) offers a high-resolution anatomical representation of veins and their relationships with lesions. Doppler modalities (color, power, spectral) allow the study of blood flow and identification of vessels to distinguish their functional characteristics. Contrast-enhanced US allows one to perform real-time angiosonography showing both the functional and the anatomical aspects of vessels.

In this technical report, the authors demonstrate the different applications of multimodal ioUS in neurosurgery for identifying the anatomical and functional characteristics of the venous compartment. They discuss the general principles and technical nuances of ioUS and analyze their potential implications for the study of various venous districts during neurosurgical procedures.
\end{abstract}

https://thejns.org/doi/abs/10.3171/2018.4.FOCUS18101

KEYWORDS intraoperative imaging; contrast-enhanced ultrasound; intraoperative ultrasound; cerebral veins; extracranial veins; dural sinuses

$\mathrm{K}$ NOWLEDGE of the venous functional anatomy is essential for the safe and effective execution of neurosurgical operations. The most critical issues to understand are the contribution of each venous structure to the drainage of each brain compartment and the dynamic nature of this behavior in pathological conditions. ${ }^{9,23}$ In cases such as tumors and vascular malformations, the physiological architecture of the venous compartment can be subverted with the creation of reversible shunts, anastomoses, and collaterals and, in some cases, even completely replacing the normal angioarchitecture. ${ }^{4}$ The highly dy- namic nature of the cerebral veins' response to head positioning and mechanical stimuli and their reorganization under different conditions (tumor presence, bone and tumor decompression, shunts, patient positioning) highlight the need for a real-time intraoperative imaging modality able to identify dynamic venous modifications.

Intraoperative ultrasound (ioUS) can provide useful real-time information in planning the management of vessels, notably venous structures. It is a rapid tomographic modality not requiring any changes in patient positioning or the intraoperative setting, while at the same time of-

ABBREVIATIONS AVM = arteriovenous malformation; CEUS = contrast-enhanced ultrasound; DAVF = dural arteriovenous fistula; GBM = glioblastoma; IJV = internal jugular vein; ioUS = intraoperative ultrasound. 
TABLE 1. Technical principles of ioUS

\begin{tabular}{ll}
\hline & Applications/Characteristics \\
\hline Probe selection & \\
\hline Linear (high frequency) & Study of superficial structures (higher definition/lower penetration) \\
\hline Convex/linear (low frequency) & Study of deep structures (lower definition/deeper penetration) \\
\hline US mode & \\
\hline B-mode (grayscale) & Initial evaluation, orientation, structure (including vessel), \& lesion detection \\
\hline Doppler (echo color \& power) & Vessel identification, flow direction, \& characterization (arterial/venous) \\
\hline CEUS & $\begin{array}{c}\text { Enhanced vessel identification (both arterial \& venous), lesion \& structure characterization } \\
\text { (arterial, venous, \& late phase) }\end{array}$ \\
\hline
\end{tabular}

fering significant spatial and temporal resolution..$^{18}$ Moreover, it provides multiple imaging modalities. The B-mode (brightness mode) has a spatial resolution comparable to that of preoperative volumetric MRI, thus allowing the study of anatomical details in real-time, given its incomparable temporal resolution. ${ }^{18}$ Power Doppler permits vessel localization relying on blood flow. Color Doppler can be used to assess flow direction in a region sensitive to flow velocity, although it is limited by the insonation angle. Spectral Doppler permits the quantification of flow velocity and its representation in waveform. Contrastenhanced ultrasound (CEUS) represents the most specific and sensitive US technique to describe blood flow in arterial, capillary, and venous districts through the use of an intravascular contrast agent to enhance backscattering from the vessels and to suppress the signal from the remaining part of tissues, thus permitting angiosonography. Its use in neurosurgery has been recently included in the European Federation of Societies for Ultrasound in Medicine and Biology (EFSUMB) guidelines. ${ }^{13,16-18,20}$

In this paper, we provide technical background on which modalities to apply when evaluating the venous circulation in neurosurgical procedures, and we review different clinical scenarios, demonstrating how the combination of different US modalities has the potential to detect all those dynamic changes that take place within the venous system during cranial surgery, and discuss the importance of the assessment of the extracranial venous system and its impact on cerebral venous drainage.

\section{Multimodal ioUS and Applications for Real-Time Venous Imaging and Management: Technical Principles}

The operator should have received specific training in the use of ioUS; therefore, the general steps in the use of US are not discussed herein. Table 1 summarizes the various US modalities used in general practice and their neurosurgical applications specifically in terms of vein visualization and management. The latter requires not only a basic theoretical knowledge of US other than the B-mode, which is provided by educational courses, but also, as already established for other organs, supervised training regarding normal and pathological US findings of brain diseases. ${ }^{12}$

The US probe and parameters should be chosen according to the lesion type, dimensions, and field depth and, in general, the information that has been collected about the area of interest. Multiple probes can be used and changed during the same intervention. Smaller probes can be used to fit smaller craniotomies or resection cavities, but they have lower resolution. Usually the entire evaluation can be performed with multifrequency linear probes with frequencies ranging from 5 to $18 \mathrm{MHz} .^{18}$

The main parameters to be set are depth and frequency. In adjusting the depth, it is possible to have a shallower or deeper image. With greater depths the image will be smaller, whereas with reduced depths the image becomes larger, although the spatial resolution will be decreased. Frequency changes allow one to modulate the spatial resolution: the higher the frequency, the higher the spatial resolution and the lower the penetration of the US beam. ${ }^{18}$

Patient positioning is crucial: the head should be elevated as compared to the thorax, the abdomen should be free, and the neck should be as supple as possible in order not to obstruct the jugular veins and the vertebral plexuses, thus facilitating venous outflow. ${ }^{10,26}$ From an imaging standpoint, the surgical field should be as parallel as possible to the floor to facilitate acoustic coupling between the probe and the organ..$^{18}$

The first part of the evaluation is performed using the $\mathrm{B}$-mode. It is conducted in two orthogonal planes, tilting and shifting the probe to visualize the entire lesion and its anatomical relationships with major landmarks (dural structures, sinuses, ventricles, choroid plexuses, and arachnoidal folds). Frequency is set to permit the maximum resolution possible that is compatible with the depth needed. ${ }^{18}$ Care should be taken not to apply too much pressure on the structures as veins are easily compressible and the simple application of the probe can modify their caliber.

The power Doppler mode is typically used to visualize vessels as it has high sensitivity and is less dependent from the insonation angle, ${ }^{18}$ whereas color Doppler and spectral Doppler should be used to study flow velocity, direction, and waveform. ${ }^{18}$ Doppler parameters should be set according to the depth and size of the vessels, speed of the flow, and angle of insonation.

The use of CEUS has been described in other papers from our group. ${ }^{13-20}$ In general, the US contrast agent is prepared and injected through a peripheral vein. Different vascular phases are analyzed: basal, arterial, peak, parenchymal, and venous. Functional characteristics are derived from the intensity of vessel scattering, the direc- 


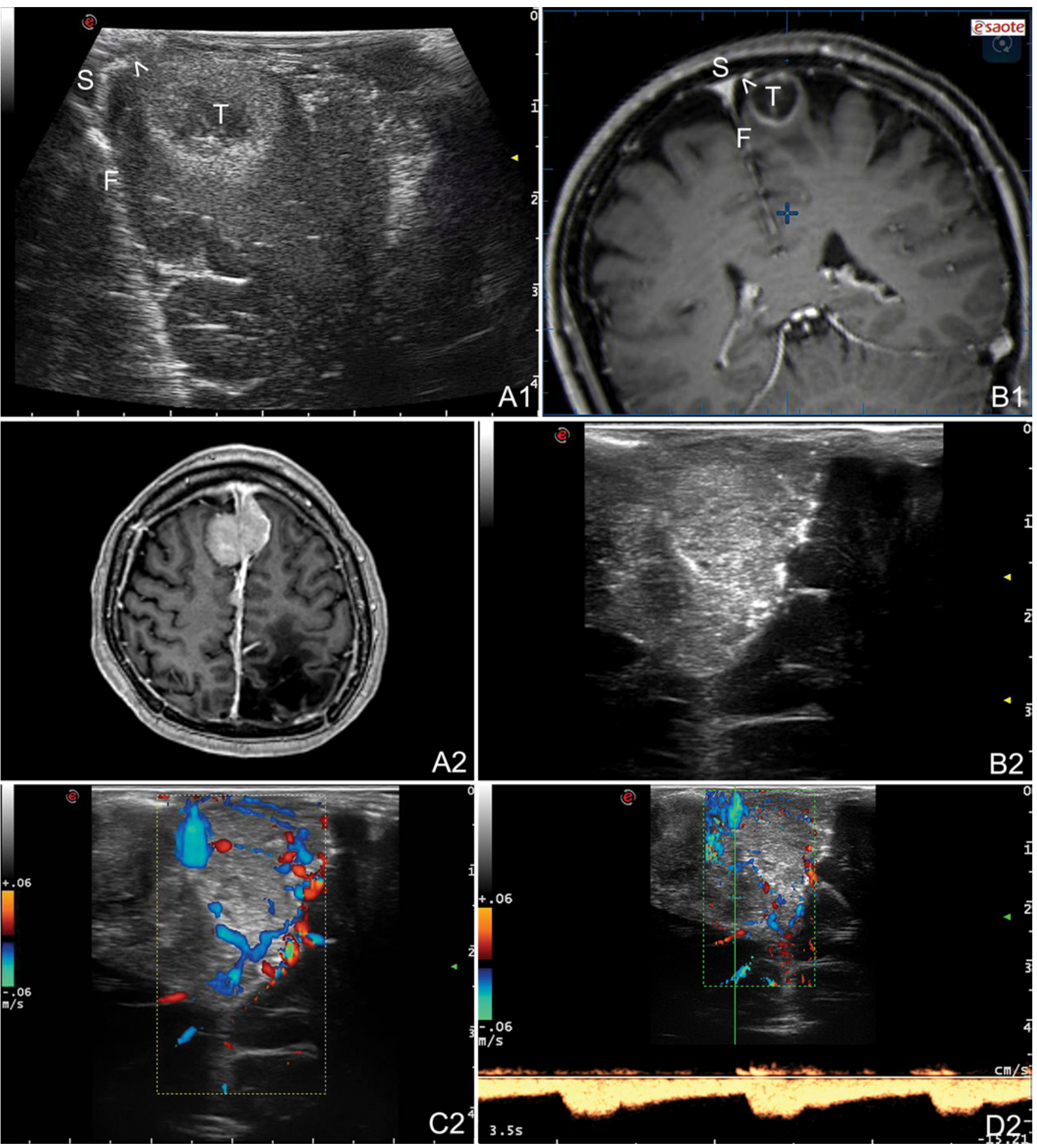

FIG. 1. Transdural multimodal US allows one to characterize the venous functional anatomy. Right frontal parasagittal meningioma (A1 and B1): ioUS permits one to tailor the dural opening, sparing the superior sagittal sinus and bridging veins. Arrowheads indicate the sinus lateral limit. Bilateral frontal sagittal meningioma (A2-D2): on preoperative MRI (A2), the superior sagittal sinus appears closed. With B-mode imaging (B2), one is unable to evaluate the patency of the sinus. Color Doppler imaging (C2) demonstrates relevant flow in the sinus, bridging veins, and collateral venous drainage. Spectral Doppler imaging (D2) allows one to study flow in the superior sagittal sinus. $F=$ falx; $S=$ sinus; $T=$ tumor. 
TABLE 2. Clinical applications of ioUS

\begin{tabular}{|c|c|c|}
\hline Application & ioUS Technique & Surgical Implication \\
\hline $\begin{array}{l}\text { Management of dural sinuses \& superficial } \\
\text { veins }\end{array}$ & $\begin{array}{l}\text { B-mode, power Doppler, color } \\
\text { Doppler, spectral Doppler, CEUS }\end{array}$ & $\begin{array}{l}\text { Venous structures localization \& morphological assessment to aid } \\
\text { in dural opening \& arachnoidal dissection }\end{array}$ \\
\hline $\begin{array}{l}\text { Management of basal dural sinuses \& } \\
\text { basal bridging veins }\end{array}$ & $\begin{array}{l}\text { Power Doppler, color Doppler, } \\
\text { spectral Doppler, CEUS }\end{array}$ & $\begin{array}{l}\text { Venous structures localization \& morphological assessment influ- } \\
\text { ences the surgical corridor \& dural opening }\end{array}$ \\
\hline Management of deep venous circulation & $\begin{array}{l}\text { Power Doppler, color Doppler, } \\
\text { spectral Doppler, CEUS }\end{array}$ & $\begin{array}{l}\text { Visualization of disrupted anatomy of venous system \& its changes } \\
\text { during surgery prior \&/or after dissection \& targeting of the lesion }\end{array}$ \\
\hline $\begin{array}{l}\text { Assessment of intraop dynamic change in } \\
\text { venous circulation }\end{array}$ & Power Doppler, CEUS & $\begin{array}{l}\text { Comprehension of specific functional anatomy of the vascular tree } \\
\text { in different lesions \& its changes during surgery }\end{array}$ \\
\hline $\begin{array}{l}\text { Assessment of changes in brain perfusion } \\
\text { associated w/ venous drainage }\end{array}$ & CEUS & $\begin{array}{l}\text { Evaluation of parenchymal blood flow in different vascular phases, } \\
\text { including the venous phase, to evaluate brain perfusion }\end{array}$ \\
\hline $\begin{array}{l}\text { Assessment of extracranial venous drain- } \\
\text { age \& implications for neurosurgical } \\
\text { procedures }\end{array}$ & Color Doppler, spectral Doppler & $\begin{array}{l}\text { Extracranial venous circulation (jugular \& vertebral veins) during } \\
\text { surgery planning \& patient positioning }\end{array}$ \\
\hline
\end{tabular}

tion and velocity of bubbles and vessels, enhancement of the lesion, time to peak enhancement, and duration of the parenchymal phase. Anatomical features are derived by observing the flow of bubbles and the vessel-enhancement position with regard to the lesion..$^{17,18}$ The examination can be repeated several times to understand the functional vascular anatomy.

In the sections that follow, we describe the use of multimodal ioUS in various cases and scenarios, highlighting the technical nuances of its application in neurosurgery.

\section{Multimodal ioUS Applications for Venous Imaging and Management: Clinical Scenarios Management of the Dural Sinuses and Superficial Veins}

Dural venous sinuses have great variability among patients with potential extension several centimeters from the midline (e.g., venous lacunae), ${ }^{3}$ whereas bridging veins can join the dura at great distances from the sinus edges before entering into it. The exact location of venous structures is extremely difficult to assess before opening the dura, thus leading to the risk of inadvertent damage. ${ }^{23}$

A B-mode US scan before dural opening allows the surgeon to identify the anatomical location and morphology of vessels, sinuses, and dural folds. Both CEUS and Doppler allow one to characterize the functional aspects of various vessels, differentiating high versus low flow, direction of the flow, flow speed, and vessel dimensions. Furthermore, the possibility of visualizing the lesion (intra- or extraaxial) together with the vessels allows one to tailor the dural opening to the intraoperative anatomy (Fig. 1 and Table 2). This is particularly helpful in cases such as parasagittal meningiomas. ${ }^{5,9,23}$ Multimodal ioUS permits one to assess the position of the lesion and the venous bridging vessels, revealing the most suitable surgical corridor and thus allowing one to tailor the durotomy accordingly.

Furthermore, in most cases in which meningioma invades or is close to the sinus, a collateral drainage system can develop, enveloping the lesion or impeding the surgical corridor. ${ }^{4,9,23}$ Preoperative workup should include an angiographic study to assess the patency of the superior sagittal sinus; $;^{9,22-25}$ however, because the study is performed while the patient is supine with a closed skull, it does not always reflect intraoperative findings. ${ }^{1,2,27}$ In some cases, such as in the parasagittal meningioma featured in Fig. 1, it must be noted that, despite the appearance of an occluded sinus on preoperative imaging, venous flow was restored intraoperatively after bone flap removal, as demonstrated by Doppler and CEUS examinations (Fig. 1 and Table 2). In these cases, the sinus should be preserved after tumor debulking because it can regain its functionality.

\section{Management of Basal Dural Sinuses and Basal Bridging Veins}

In subtemporal, pretemporal, or transsylvian approaches, retraction of the temporal lobe or access to the opticocarotid region is commonly restricted by bridging veins toward the basal sinuses.? In this setting, vein sparing is mandatory. An ioUS scan before dural opening, as well as during resection, permits one to visualize the bridging veins without the need for direct exposure. In particular Doppler modalities, especially those performed in combination with CEUS, one can visualize all vessels including those at different depths within the field of view, both arterial and venous, independently of the angle of insonation (Fig. 2 and Table 2). This feature can influence surgical decision-making as regards the better corridor to reach the target and to open the dura accordingly.

\section{Management of Deep Venous Circulation}

When dealing with lesions close to the deep venous system, it is important to visualize the entire vascular tree and how it is distorted by tumor. Even if not properly encased by the lesion, the deep venous system represents a critical aspect of tumor debulking and dissection. The B-mode coupled with vascular imaging via Doppler or CEUS allows one to visualize the lesion to study its vascularity as well as the disrupted anatomy of the venous system and can be repeated several times, permitting one to follow its changes during various phases of the surgery. In particular, CEUS is useful as it allows one to perform 


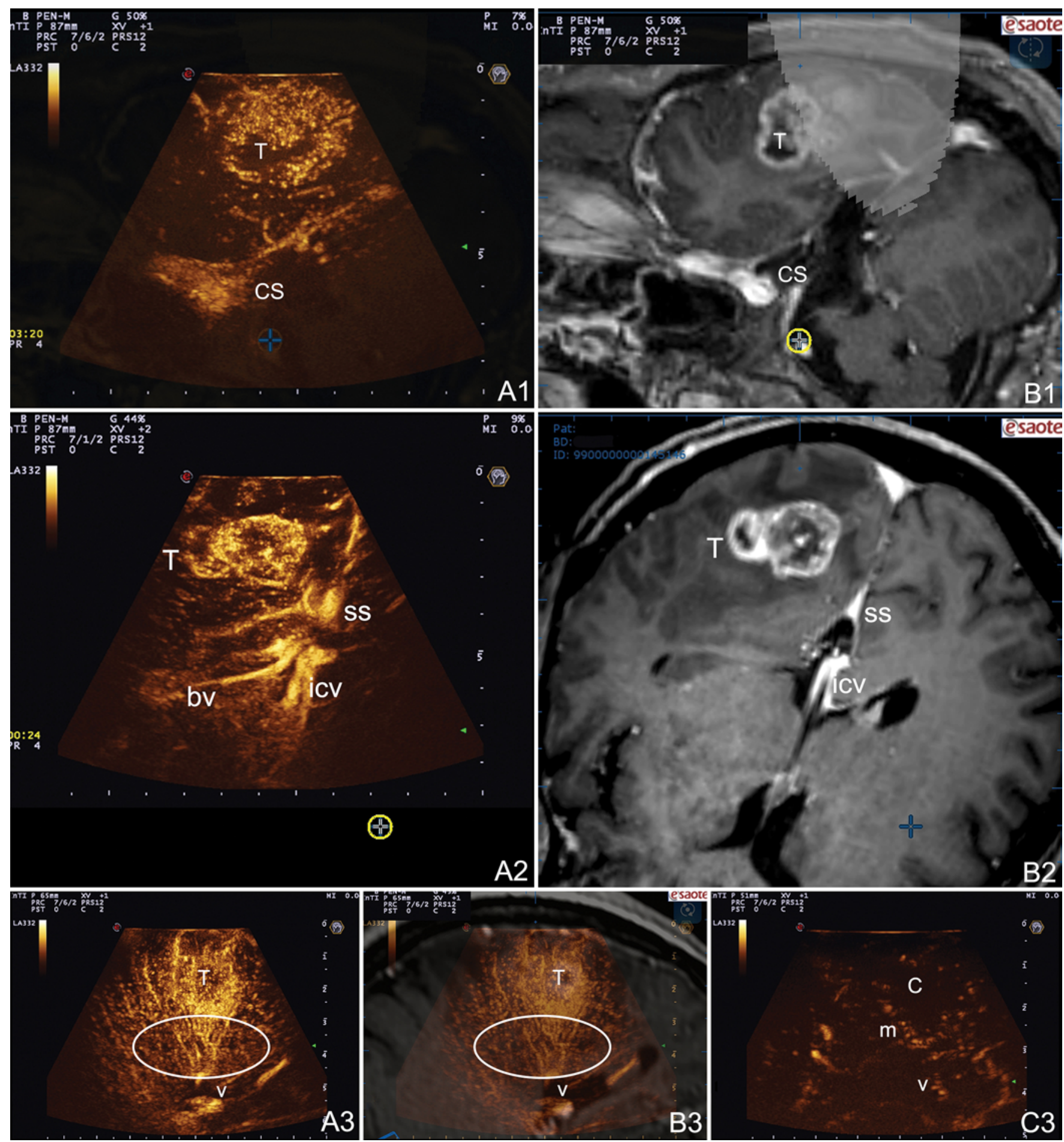

FIG. 2. Intraoperative multimodal US permits one to visualize and characterize the entire vascular venous tree and its changes during surgery. A case of right temporal GBM allows for the study of both arterial and venous relationships; the cavernous sinus is visualized together with the basal venous tributaries on navigated CEUS (A1) and the corresponding coplanar preoperative MRI (B1). A case of right parietooccipital GBM: a CEUS scan (A2) and the corresponding coplanar preoperative gadolinium-enhanced MR image (B2). A case of left parietooccipital GBM (A3-C3): CEUS highlights tumor together with venous, capillary, and arterial vessels and permits the study of deep veins up to a depth of $9 \mathrm{~cm}$ from the surface, allowing their identification in the deeper part of the lesion or in cases of deep-seated lesions. Pre-resection CEUS examinations highlight venous drainage through small vessels directed toward the ventricle (ellipses, A3 and B3). After complete resection, venous drainage disappeared $(\mathbf{C} 3)$. bv = basal vein; $C=$ cavity; $C S=$ cavernous sinus; icv = internal cerebral veins; $m=$ margins; $s s=$ straight sinus; $T=$ tumor; $v=$ ventricle. 


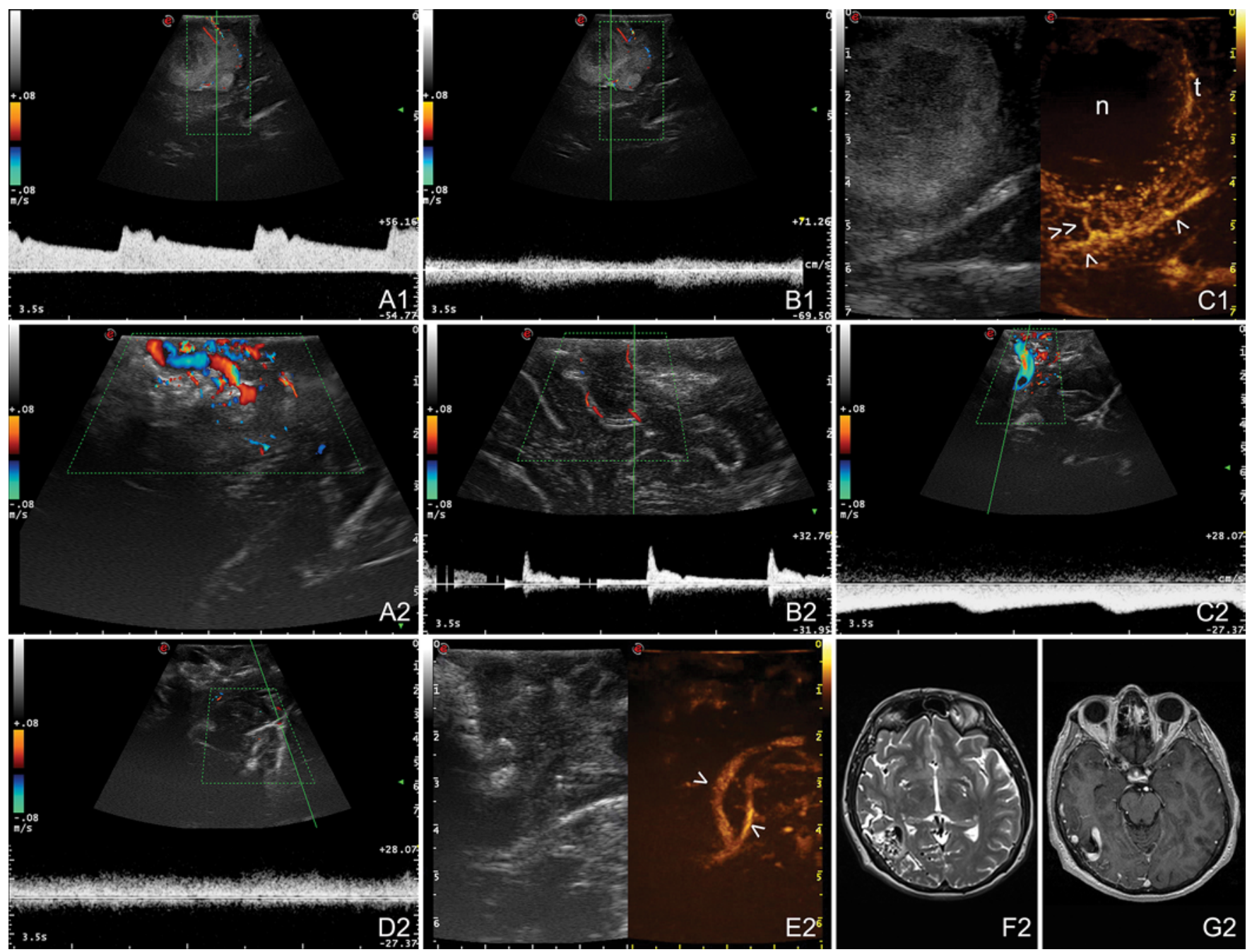

FIG. 3. Intraoperative multimodal US to characterize vascular functional anatomy of the lesion and surrounding vessels both on the surface and in depth. Right temporoparietal GBM (A1-C1): spectral Doppler demonstrates that the superficial vessel is an artery (A1) and the deeper vessel is a vein (B1); note the typical flow patterns. CEUS (C1) permits characterization of both tumor and surrounding vessels: in particular, the deep vein is part of the venous drainage of the lesion. Arrowheads indicate the vein visualized in B1; double arrowhead, the radial venous drainage toward the vein. Right parietooccipital AVM (A2-G2): color Doppler permits visualization of the AVM nidus, providing an overview of the malformation (A2). Spectral Doppler permits identification and study of each component of the AVM: arterial feeder (B2), venous enlargement (C2), and straight sinus (D2). Note the different flow patterns with different shapes and flow velocity for each vascular structure. CEUS (E2) highlights deep venous drainage toward the straight sinus (arrowheads). Preoperative T2-weighted (F2) and gadolinium-enhanced (G2) MRI studies. $n=$ necrosis; $t$ = viable tumor.

real-time angiosonography to visualize both the arteries and the veins simultaneously, even deep, small low-flow vessels (Fig. 2 and Table 2). The images are generated by the machine's reliance only on harmonic signals produced by intravascular microbubbles. This is particularly useful when dealing with deep-seated structures because the signal generated by the parenchyma is subtracted from the images, as in digital subtraction angiography, and only the vascular tree is displayed with great specificity and detail.

\section{Assessment of Intraoperative Dynamic Changes in Venous Circulation}

The different real-time dynamic modalities of ioUS can not only integrate the information provided by preopera- tive imaging but can also detect and highlight changes that occur during surgery, from bone flap removal with the expansion of compressed veins to flow normalization in arterialized veins and the disappearance of arteriovenous shunts.

Intra- and extraaxial lesions display different patterns of venous drainage that can be studied before and after tumor removal, aiding in distinguishing between tumorrelated veins and physiological veins (Figs. 1-3 and Table 2). Intraaxial tumors, such as glioblastoma (GBM), represent a sort of shunt with centripetal drainage toward the ependymal veins of the ventricles through hypertrophic medullary veins. After tumor removal, these hypertrophic medullary veins were no longer visible (Fig. 2). ${ }^{19}$ 

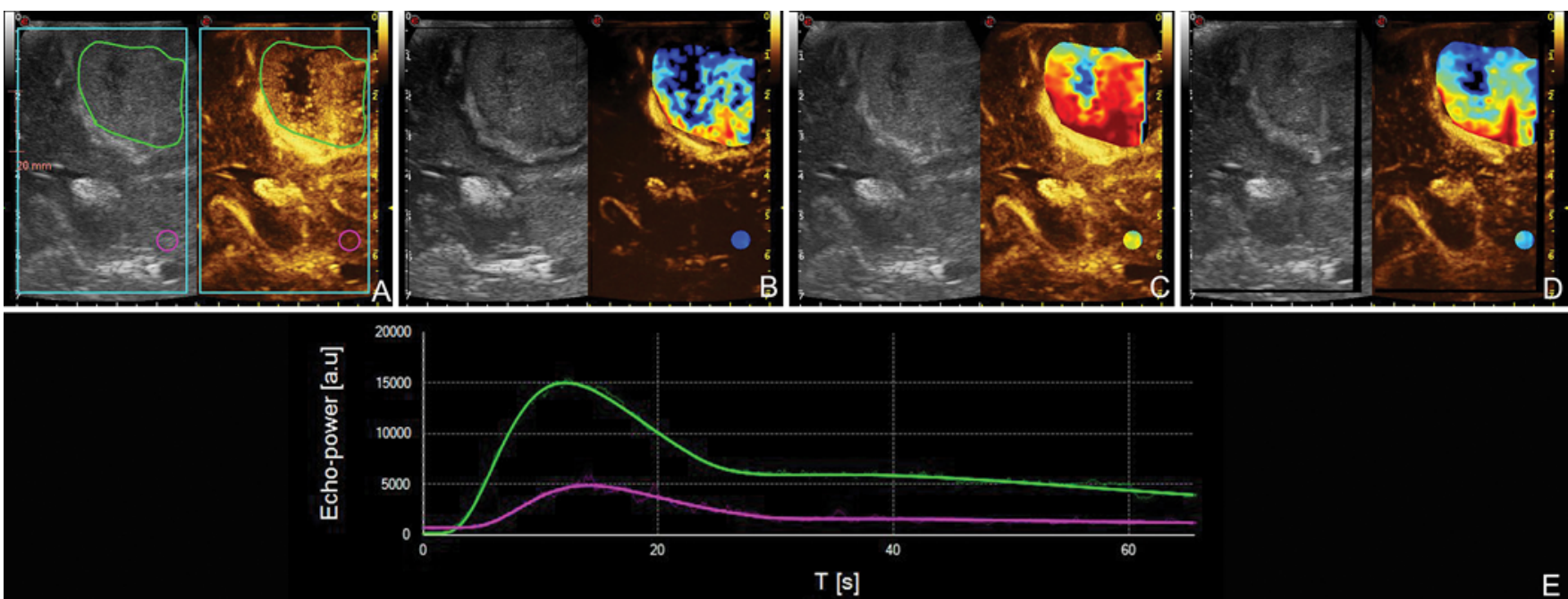

\begin{abstract}
FIG. 4. CEUS perfusion analysis in a case of left frontal GBM. CEUS tumor intensity (green) is normalized to a region of interest (ROI; purple) placed on healthy parenchyma (A): arterial phase (B), peak phase (C), and venous phase (D). The clip featured in the time-intensity curve (E) was obtained during a 40-second registration, performed while keeping the probe still and processed using proprietary software for quantitative imaging (VueBox). Postprocedural analysis is conducted by analyzing different ROls manually drawn along tumor borders, major vessels, and lobar parenchyma (tumor, green; healthy parenchyma, purple).
\end{abstract}

In extraaxial tumors, such as meningiomas, drainage is mediated by several subarachnoid veins that can also be confused with healthy brain vessels (Fig. 1). ${ }^{15}$ After tumor debulking, these veins were usually no longer significant.

In surgery for arteriovenous malformation (AVM) and dural arteriovenous fistula (DAVF), a critical aspect is recognizing the flow architecture in order to approach the lesion. ${ }^{14,16}$ CEUS allows one to visualize the direction of the flow and different components of the malformation without having to directly expose the structures prior to dural opening (Fig. 3 and Table 2). In cases of DAVF, CEUS permits one to study the arterial feeder and the arterialized veins in great detail. This feature can be decisive in cases of spinal DAVF in which a congested venous plexus can hide the fistula. ${ }^{16}$ Furthermore, the possibility of obtaining an ioUS scan after bone removal allows the surgeon to open the dura according to the malformation anatomy. Because US imaging presents a sectorial tomographic scan, it allows one to observe the whole surgical field, from the surface to the depth, without the need to directly expose all structures involved. It is also important to obtain multiple scans at different time points during the surgical procedure in order to assess the changes occurring over time.

\section{Characterization of Brain Perfusion Changes Associated With Venous Drainage}

Cerebral veins and sinuses are mainly viewed as hydraulic collectors, driving blood out of the head back to the heart. However, their role has larger implications, regulating parenchymal drainage and cerebrospinal fluid homeostasis and in general influencing intracranial pressure and brain perfusion. ${ }^{5,6,10,26,28}$ Rapid alterations of the venous system such as those occurring during surgery are implicated in localized parenchymal drainage altera- tion with subsequent venous engorgement, local hypertension, parenchymal perfusion impairment, and eventually infarction. ${ }^{23}$ CEUS allows one to analyze tissue perfusion. ${ }^{17,18}$ Microbubble quantification is performed using off-line dedicated software, providing information about parenchymal and lesion perfusion with multiparametric representations of time-intensity curves, as described in Fig. 4, and measuring the echo intensity enhancement in a given period of time in the selected region of interest. This allows one to quantitatively evaluate the flow in different vascular phases, including the venous phase, and thus indirectly assessing brain perfusion.

\section{Evaluation of the Extracranial Venous Drainage and Implications for Neurosurgical Procedures}

Cerebral venous outflow has been extensively studied in recent years, and it has been correlated mainly to other CNS pathologies such as idiopathic intracranial hypertension, cerebrospinal venous insufficiency, and multiple sclerosis. ${ }^{8,11}$

Cephalic venous drainage is guaranteed by two major systems-internal jugular veins (IJVs) and vertebral venous plexuses-representing an anastomotic drainage system between the extracranial and intracranial compartments. ${ }^{26}$ The supine position favors cerebral venous flow through the IJV, while in the orthostatic position blood outflow is mainly redirected to the vertebral and azygos veins ${ }^{6,26}$ Furthermore, in about $85 \%$ of subjects, IJV valves are present as phylogenetic remnants at the junction of the brachiocephalic trunk, potentially causing retrograde flow during the Valsalva maneuver. ${ }^{21}$

These positional modifications highlight the need to take into account the position of the head relative to the neck and the rest of the body in the operating room. It is necessary to understand the real venous functional anatomy, which cannot be demonstrated by preoperative stand- 


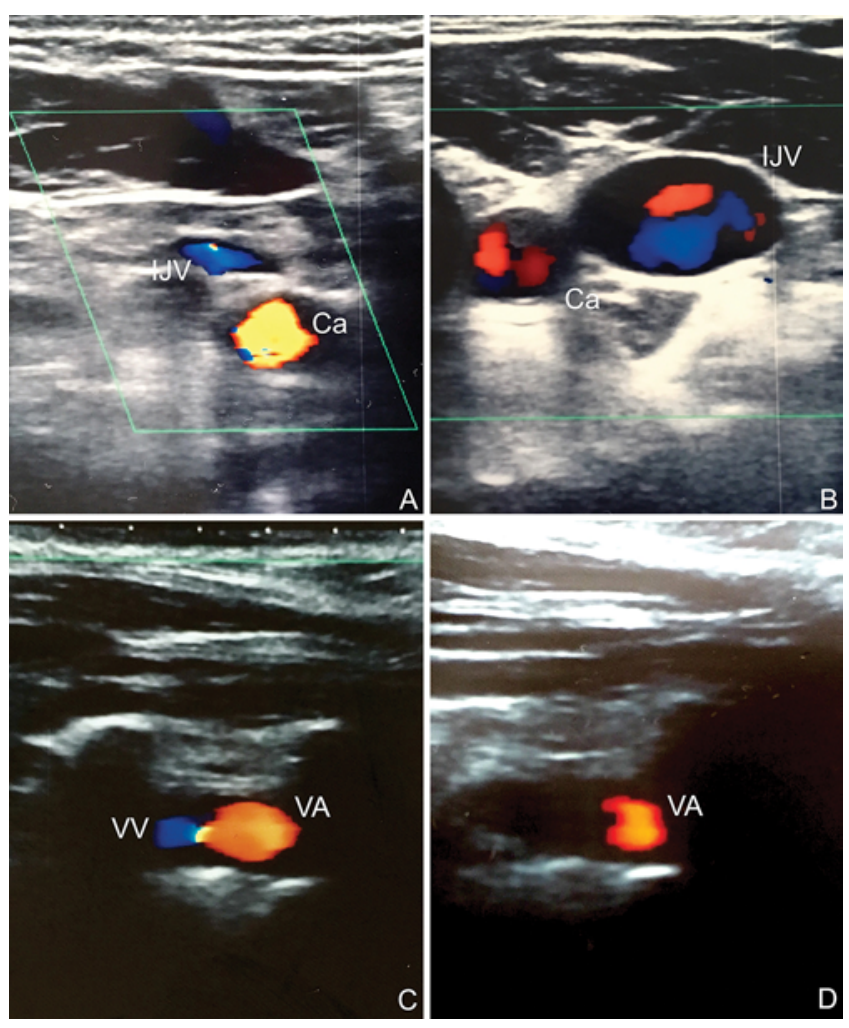

FIG. 5. Cerebral venous outflow. Area and flux direction in the IJV in a patient sitting (A) or supine (B). Transverse cervical color Doppler at the thyroid level showing the IJV and carotid artery $(\mathrm{Ca})$; note the change in the IJV area when the patient is supine or sitting. Flux direction in the vertebral venous plexus (VV) in a patient sitting (C) or supine (D). Longitudinal cervical color Doppler at the level of the carotid bifurcation showing the VV and vertebral artery (VA); note the change in flux in the VV when the patient is sitting or supine. In the latter position, flow in the VV disappears.

ard imaging as it is usually performed in a supine patient with the head in a neutral position.

Venous outflow should be assessed during the preoperative workup to visualize postural changes as described in Fig. 5. The combination of morphological and hemodynamic parameters of color Doppler represents an ideal direct, real-time method for the study of hemodynamics of cerebral venous outflow.

The principal significance for neurosurgery is the objective need to consider extracranial venous outflow during surgical planning. Poor positioning of the patient with the head too rotated or flexed, lower than the chest, or with compression of the abdomen could lead to insufficient venous drainage with a consequent increase in intracranial pressure, brain swelling, and venous bleeding and less compliance if vein sacrifice is needed.

\section{Conclusions}

ioUS permits real-time, dynamic intraoperative characterization of the venous compartment not limited to the anatomical representation via the B-mode, but through multiple modalities allowing for the evaluation of blood flow, tissue and tumor perfusion, changes in venous dis- tricts, and extracranial outflow. The main limitations of ioUS are its operator dependency and steep learning curve. As with surgery, ioUS is a technique that requires training and practice in order to fully understand its potential and to develop the ability to tailor and maximize its response to a patient's clinical and surgical needs. However, multimodal ioUS is a great tool, used alone or in a synergistic manner with other imaging modalities, in the surgical armamentarium.

\section{References}

1. Ayanzen RH, Bird CR, Keller PJ, McCully FJ, Theobald MR, Heiserman JE: Cerebral MR venography: normal anatomy and potential diagnostic pitfalls. AJNR Am J Neuroradiol 21:74-78, 2000

2. Bozzao A, Finocchi V, Romano A, Ferrante M, Fasoli F, Trillò G, et al: Role of contrast-enhanced MR venography in the preoperative evaluation of parasagittal meningiomas. Eur Radiol 15:1790-1796, 2005

3. Brockmann C, Kunze SC, Schmiedek P, Groden C, Scharf J: Variations of the superior sagittal sinus and bridging veins in human dissections and computed tomography venography. Clin Imaging 36:85-89, 2012

4. Debernardi A, Quilici L, La Camera A, Boccardi E, Cenzato M: Torcular meningioma with multi-venous sinus invasion: compensatory drainage veins and surgical strategy. World Neurosurg 109:451-454, 2018

5. Ferroli P, Acerbi F, Tringali G, Albanese E, Broggi M, Franzini A, et al: Venous sacrifice in neurosurgery: new insights from venous indocyanine green videoangiography. J Neurosurg 115:18-23, 2011

6. Gisolf J, van Lieshout JJ, van Heusden K, Pott F, Stok WJ, Karemaker JM: Human cerebral venous outflow pathway depends on posture and central venous pressure. J Physiol 560:317-327, 2004

7. Han H, Deng X, Fong AH, Zhang M: Dural entrance of the bridging vein in the middle cranial fossa: a novel classification of the cerebral veins for preoperative planning. Neurosurgery 67 (3 Suppl Operative):ons9-ons15, 2010

8. Kugler N, Patel PJ, Lee CJ: Chronic cerebrospinal venous insufficiency in multiple sclerosis: a failed concept. Vasc Spec Int 31:11-14, 2015

9. Mathiesen T, Pettersson-Segerlind J, Kihlström L, Ulfarsson E: Meningiomas engaging major venous sinuses. World Neurosurg 81:116-124, 2014

10. Mavrocordatos P, Bissonnette B, Ravussin P: Effects of neck position and head elevation on intracranial pressure in anaesthetized neurosurgical patients: preliminary results. J Neurosurg Anesthesiol 12:10-14, 2000

11. Nedelmann M, Kaps M, Mueller-Forell W: Venous obstruction and jugular valve insufficiency in idiopathic intracranial hypertension. J Neurol 256:964-969, 2009

12. Perin A, Prada FU, Moraldo M, Schiappacasse A, Galbiati TF, Gambatesa E, et al: USim: A new device and app for case-specific, intraoperative ultrasound simulation and rehearsal in neurosurgery. A preliminary study. Oper Neurosurg (Hagerstown) 14:572-578, 2018

13. Prada F, Del Bene M, Casali C, Saladino A, Legnani FG, Perin A, et al: Intraoperative navigated angiosonography for skull base tumor surgery. World Neurosurg 84:1699-1707, 2015

14. Prada F, Del Bene M, Faragò G, DiMeco F: Spinal dural arteriovenous fistula: is there a role for intraoperative contrastenhanced ultrasound? World Neurosurg 100:712.e15-712. e18, 2017

15. Prada F, Del Bene M, Moiraghi A, Casali C, Legnani FG, Saladino A, et al: From grey scale B-mode to elastosonog- 
raphy: multimodal ultrasound imaging in meningioma surgery-pictorial essay and literature review. BioMed Res Int 2015:925729, 2015

16. Prada F, Del Bene M, Saini M, Ferroli P, DiMeco F: Intraoperative cerebral angiosonography with ultrasound contrast agents: how I do it. Acta Neurochir (Wien) 157:1025-1029, 2015

17. Prada F, Perin A, Martegani A, Aiani L, Solbiati L, Lamperti M, et al: Intraoperative contrast-enhanced ultrasound for brain tumor surgery. Neurosurgery 74:542-552, 2014

18. Prada F, Solbiati L, Martegani A, DiMeco F (eds): Intraoperative Ultrasound (IOUS) in Neurosurgery: from Standard B-mode to Elastosonography. Cham, Switzerland: Springer, 2016

19. Prada F, Vitale V, Del Bene M, Boffano C, Sconfienza LM, Pinzi V, et al: Contrast-enhanced MR imaging versus contrast-enhanced US: a comparison in glioblastoma surgery by using intraoperative fusion imaging. Radiology 285:242249, 2017

20. Sidhu PS, Cantisani V, Dietrich CF, Gilja OH, Saftoiu A, Bartels E, et al: The EFSUMB Guidelines and Recommendations for the Clinical Practice of Contrast-Enhanced Ultrasound (CEUS) in Non-Hepatic Applications: Update 2017 (long version). Ultraschall Med 39:e2-e44, 2018

21. Silva MA, Deen KI, Fernando DJ, Sheriffdeen AH: The internal jugular vein valve may have a significant role in the prevention of venous reflux: evidence from live and cadaveric human subjects. Clin Physiol Funct Imaging 22:202-205, 2002

22. Sindou M: Meningiomas involving major dural sinuses: should we attempt at radical removal and venous repair? World Neurosurg 81:46-47, 2014

23. Sindou M, Auque J, Jouanneau E: Neurosurgery and the intracranial venous system. Acta Neurochir Suppl 94:167175,2005

24. Sindou M, Hallacq P: Venous reconstruction in surgery of meningiomas invading the sagittal and transverse sinuses. Skull Base Surg 8:57-64, 1998

25. Sindou MP, Alvernia JE: Results of attempted radical tumor removal and venous repair in 100 consecutive meningiomas involving the major dural sinuses. J Neurosurg 105:514-525, 2006

26. Valdueza JM, von Münster T, Hoffman O, Schreiber S, Einhäupl KM: Postural dependency of the cerebral venous outflow. Lancet 355:200-201, 2000

27. Wetzel SG, Kirsch E, Stock KW, Kolbe M, Kaim A, Radue EW: Cerebral veins: comparative study of CT venography with intraarterial digital subtraction angiography. AJNR Am J Neuroradiol 20:249-255, 1999

28. Wilson MH: Monro-Kellie 2.0: The dynamic vascular and venous pathophysiological components of intracranial pressure. J Cereb Blood Flow Metab 36:1338-1350, 2016

\section{Disclosures}

Dr. Mauri has been a consultant for Elesta SrL.

\section{Author Contributions}

Conception and design: Prada, Del Bene. Acquisition of data: Prada, Richetta, Saini, Santuari. Analysis and interpretation of data: Prada, Del Bene, Mauri, Lamperti, Richetta, Santuari. Drafting the article: Prada, Del Bene, Lamperti, Vailati, Santuari. Critically revising the article: Prada, Del Bene, Mauri, Lamperti, Vailati, Saini, Santuari, Kalani, DiMeco. Reviewed submitted version of manuscript: Prada, Del Bene, Mauri, Santuari, Kalani, DiMeco. Study supervision: Prada, DiMeco.

\section{Correspondence}

Francesco Prada: Fondazione IRCCS Istituto Neurologico C. Besta, Milan, Italy. francesco.prada@istituto-besta.it. 